\title{
A THEOREM ON POWER SERIES WHOSE COEFFICIENTS HAVE GIVEN SIGNS
}

\author{
W. H. J. FUCHS ${ }^{1}$
}

1. The following theorem, first proved by A. Hurwitz and G. Pólya, is well known ([3] or [1, p. 99]).

If $\sum_{k=0}^{\infty} a_{k} z^{k}$ is a power series of finite radius of convergence, then it is possible to find a sequence $\left\{\epsilon_{k}\right\}\left(\epsilon_{k}= \pm 1\right)$ such that the series $\sum_{k=0}^{\infty} \epsilon_{k} a_{k} z^{k}$ has the circle of convergence as natural boundary.

In this note I prove the following companion-piece to Pólya's theorem.

THEOREM. If $\left\{\epsilon_{k}\right\}_{k=0}^{\infty}$ is a sequence with $\epsilon_{k}= \pm 1$, then there is always $a$ power series $\sum a_{k} z^{k}, a_{k}>0$, of finite radius of convergence such that the series $\sum \epsilon_{k} a_{k} z^{k}$ can be analytically continued across a semi-circle on its circle of convergence.

This theorem answers in the negative the question: Is there a "universal scrambling sequence" $\left\{\epsilon_{k}\right\}, \epsilon_{k}= \pm 1$, turning every power series $\sum a_{k} z^{k}$ with positive coefficients into a power series $\sum \epsilon_{k} a_{k} z^{k}$ having the circle of convergence as natural boundary? This problem was raised by Mrs. Turán, and I am indebted to Dr. P. Erdös for communicating it to me.

An example ( $\$ 4)$ shows that the semi-circle in the statement of the theorem can not be replaced by a larger arc.

A question which remains open is to find a corresponding theorem for the case that $\left\{\epsilon_{k}\right\}$ is a given sequence of complex numbers of absolute value one.

2. The following lemmas are required.

LemMa 1. Let $\Lambda=\left\{\lambda_{n}\right\}$ be a sequence of positive numbers no two of which are at a distance less than $c>0$ from each other. Let

$$
g(z)=\prod_{\lambda \in \Lambda} \frac{\lambda-z}{\lambda+z} e^{2 z / \lambda} .
$$

Then there are constants $A$ and $B$ such that in $x \geqq 0,|z-\lambda| \geqq c / 4(\lambda \in \Lambda)$

$$
0<\left(B e^{\phi(r)}\right)^{x} \leqq|g(z)| \leqq\left(A e^{\phi(r)}\right)^{x}
$$

where $r=|z|=|x+i y|$ and

Received by the editors April 9, 1956 and, in revised form, August 23, 1956.

1 This research was supported by the United States Air Force under Contract No. AF 18(600)-685 monitored by the Office of Scientific Research. 


$$
\phi(r)=\sum_{\lambda<r ; \lambda \in \Lambda} 2 / \lambda .
$$

For a proof of this lemma see [2, Lemmas 3 and 4].

Lemma 2. Let $M=\{\mu\}$ be a sequence of positive numbers whose mutual distances are $\geqq 1$. Suppose that the function $h(\zeta)=h(\xi+i \eta)$ is regular in the region $\xi \geqq 0, \zeta \neq 0$ except for simple poles at the points $\zeta=\mu \in M$. Suppose further that there are positive constants $A, \alpha, \beta$ $(\beta<\pi)$ such that

$$
|h(\xi)|=|h(\xi+i \eta)|<A e^{\alpha \xi-\beta|\eta|}
$$

in $\xi \geqq 0, \zeta \neq 0$, except in circles of radius $1 / 4$ with centers at the points $\mu \in M$.

Then the function

$$
H(z)=\sum_{\mu \in M} r_{\mu} z^{\mu}
$$

is regular in the sector $0<|z|,|\arg z|<\beta$; where $r_{\mu}$ is the residue of $h(\zeta)$ at $\mu$.

Proof. Let $C_{R}$ be the semicircle $|\zeta|=R, \xi \geqq 0$ and let $L_{R}$ be a curve with endpoints $\zeta=i R$ and $\zeta=-i R$ which runs along the imaginary axis except for an indentation into the right half plane near $\zeta=0$.

By the residue theorem

$$
\frac{1}{2 \pi i} \int h(\zeta) e^{-k \zeta} d \zeta=\sum_{\mu<R ; \mu \in M} r_{\mu} e^{-k \mu}
$$

where the integration is along $C_{R}+L_{R}$. If the number $k$ is chosen positive and larger than $\alpha$, then on $C_{R}$

$$
\left|h(\zeta) e^{-k \zeta}\right|<A e^{(\alpha-k) \xi-\beta|\eta|}
$$

and therefore

$$
\int_{C_{R}} h(\zeta) e^{-k \zeta} d \zeta \rightarrow 0
$$

as $R \rightarrow \infty$ through a sequence of values avoiding the intervals $|\xi-\mu|$ $<1 / 4$ on the real axis. It follows that for $z=e^{-k}(k>\alpha)$ the series

$$
\sum_{\mu \in M} r_{\mu} z^{\mu}
$$

converges to a function 


$$
H(z)=\sum r_{\mu} z^{\mu}=\frac{1}{2 \pi i} \int_{-i \infty}^{i \infty} h(\zeta) z^{\zeta} d \zeta,
$$

where the path of integration is the imaginary axis with an indentation near $\zeta=0$. For purely imaginary values of $\zeta,\left|z^{5}\right|=\left|z^{i \eta}\right|$ $=e^{-\eta \cdot \arg z}$. Hence, in $|\arg z| \leqq \beta^{\prime}<\beta, \quad\left|h(i \eta) z^{i \eta}\right|<A e^{-\beta|\eta|+|\eta| \cdot|\arg z|}$ $<A e^{-\left(\beta-\beta^{\prime}\right)|\eta|}$. This shows that the integral on the right-hand side of (2) is uniformly convergent in $|\arg z| \leqq \beta^{\prime}<\beta$. Therefore it defines the analytic continuation of $H(z)$ into the whole sector $|\arg z|<\beta$.

3. Proof of the theorem. Let $\{\lambda\}=\Lambda$ be the set of those odd multiples of $1 / 2$ for which $\epsilon_{\lambda-1 / 2} \epsilon_{\lambda+1 / 2}=-1$. Write

$$
\begin{aligned}
\phi(r) & =\sum_{\lambda<r} 2 / \lambda, \\
m & =\liminf _{r \rightarrow \infty}(\phi(r)-\log r), \\
M & =\limsup _{r \rightarrow \infty}(\phi(r)-\log r) .
\end{aligned}
$$

We consider separately the five cases:
(i) $-\infty<M<\infty$.
(ii) $M=-\infty$.
(iii) $-\infty<m<\infty$.
(iv) $m=\infty$.
(v) $m=-\infty, M=\infty$.

These cases are not mutually exclusive, but they cover all possibilities.

(i) $-\infty<M<\infty$. Define $g(z)$ by (1). By Lemma 1

$$
0<\lim \sup _{n \rightarrow \infty}|g(n)|^{1 / n} / n=C<\infty \quad(n=1,2, \cdots) .
$$

The function

$$
h(\zeta)=(C \zeta)^{-\zeta} g(\zeta) \operatorname{cosec} \pi \zeta
$$

satisfies the hypotheses of Lemma 2 with $\beta=\pi / 2, M=\{1,2,3, \cdots\}$. The residue of $h(\zeta)$ at $n$ is

$$
r_{n}=(-1)^{n} g(n) / \pi(C n)^{n} .
$$

By the choice of $C$, the series

$$
\sum r_{n}(-z)^{n}=\sum c_{n} z^{n}=f(z)
$$

has radius of convergence 1 . The sign of $c_{n}$ is the same as that of $g(n)$. But $g(x)$ changes sign between those integers $k, k+1$ for which $\epsilon_{k}$ and $\epsilon_{k+1}$ are of opposite sign and nowhere else. Hence $\epsilon_{n} g(n)$ is of constant sign. Also, by Lemma $2, f(z)$ is regular in $|\arg (-z)|<\pi / 2$, 
i.e. in $x<0$. Therefore one of the two functions $\pm f(z)$ has the required properties.

(ii) $M=-\infty$. We can find a sequence $\{\nu\}$ of odd multiples of $1 / 2$ which has no terms in common with $\Lambda$ and for which $\lim \sup \{\phi(r)$ $\left.+\sum_{\nu<r} 4 / \nu-\log r\right\}=0$, say. The construction of the previous case can now be used, if $g(\zeta)$ is replaced by

$$
g(\zeta) \cdot\left\{\Pi \frac{\nu-\zeta}{\nu+\zeta} e^{2 \zeta / \nu}\right\}^{2} .
$$

(iii) $-\infty<m<\infty$. Let $g(\zeta)$ again be defined by (1). If $D$ is any positive number, the function

$$
h(\zeta)=(D \zeta)^{\zeta} / g(\zeta)
$$

satisfies the hypotheses of Lemma 2 , with $\{\mu\}=\{\lambda\}, \beta=\pi / 2 .{ }^{2}$ The residue at $\lambda=\rho$ is

$$
r_{\rho}=(D \rho)^{\rho} / g^{\prime}(\rho)
$$

Now

$$
g^{\prime}(\rho)=-\prod_{\lambda \in \Lambda ; \lambda \neq \rho} \frac{\lambda-\rho}{\lambda+\rho} e^{2 \lambda / \rho} \cdot \frac{e^{2}}{2 \rho}
$$

and so, by Lemma $1 g^{\prime}(\rho)$ lies between

$$
\left(B_{1} e^{\phi(\rho)}\right)^{\rho} \text { and }\left(A_{1} e^{\phi(\rho)}\right)^{\rho} \text {, }
$$

where $A_{1}$ and $B_{1}$ are independent of $\rho$. Since $\lim \inf (\phi(\rho)-\log \rho)$ is finite, the constant $D$ can be adjusted so that the series

$$
\sum_{\rho \in \Lambda} r_{\rho} \cdot z^{\rho-1 / 2}=\psi(z)
$$

has radius of convergence 1 . By Lemma $2 \psi(z)$ is regular in $x>0$. The values of $\epsilon$ at successive terms of the sequence $\{\rho-1 / 2\}$ are of opposite sign, since two such integers are separated by exactly one term of the sequence $\Lambda$.

The coefficients $r_{\rho}$ of two consecutive terms in the power series are also of opposite sign, since the slope of $g(x)$ has opposite signs at successive zeros $\rho$ of $g(x)$.

Therefore $\pm \psi(z)$ satisfies all requirements, except that it has zero coefficients for all integers which are not of the form $\rho-1 / 2, \rho \in \Lambda$. By adding to $\pm \psi(z)$ an entire function whose power series has suitable signs, an example satisfying all requirements is obtained.

2 This is easily verified, if the inequality $\theta \sin \theta \leqq(\pi / 2)(1-\cos \theta)$ is noted. 
(iv) $m=\infty$. We can choose a sub-sequence of $\Lambda$ such that we have case (iii) for the new sequence and such that any two consecutive terms of the sub-sequence are separated by an even number of terms in the original sequence $\Lambda$. The construction of case 3 can now be applied.

(v) $m=-\infty, M=\infty$. Put

$$
\gamma(r)=\phi(r)-\log r=\sum_{\lambda<r} 2 / \lambda-\log r .
$$

The hypothesis $m=-\infty, M=\infty$ implies that given $q>0$ there are arbitrarily large numbers $h, k$ such that

$$
\inf _{h<r<k} \gamma(r)-\gamma(h)=-q
$$

and

$$
0 \leqq \gamma(k)-\gamma(h) \leqq 1 .
$$

A set $M=\{\mu\}$ is now constructed as follows.

First a sequence of nonoverlapping intervals $I_{1}, I_{2}, \ldots$ is chosen such that in $I_{q}=\left(h_{q}, k_{q}\right)$ (3) and (4) hold, with $h=h_{q}$ and $k=k_{q}$, and such that $h_{q+1}>10 k_{q}(q=1,2, \cdots)$. All terms of $\Lambda$ which lie in $I_{q}$ are terms of the new sequence $M$, in fact $M \cap I_{q}=\Lambda \cap I_{q}(q=1,2, \cdots)$.

If $\delta(r)=\sum_{\mu<r ; \mu \in M} 2 / \mu-\log r$, then it follows that (3) and (4) hold with $\delta$ in place of $\gamma$ and $h=h_{q}, k=k_{q}$.

Next we select further positive odd multiples of $1 / 2$ as members of $M$.

At first enough of these are chosen from the interval $\left(0, h_{1}\right)$ to make

$$
0<\delta\left(h_{1}\right) \leqq 1 .
$$

This gives, by (4),

$$
0 \leqq \delta\left(k_{1}\right) \leqq 2 .
$$

Next enough terms are added between $k_{1}$ and $h_{2}$ to make

and

$$
0 \leqq \delta(r) \leqq 2 \quad\left(k_{1} \leqq r \leqq h_{2}\right)
$$

$$
1 \leqq \delta\left(h_{2}\right) \leqq 2
$$

Then, by (3)

and we make

$$
1 \leqq \delta\left(k_{2}\right) \leqq 3
$$

$$
\begin{aligned}
& 1 \leqq \delta(r) \leqq 3 \\
& 2 \leqq \delta\left(h_{3}\right) \leqq 3 .
\end{aligned}
$$


Continuing in this way we can choose $M$ so that

$$
q-2 \leqq \delta(r) \leqq q \quad\left(k_{q-1} \leqq r \leqq h_{q} ; q=2,3, \cdots\right) .
$$

Hence $\delta(r) \rightarrow \infty$ as $r \rightarrow \infty$ through any set of values outside the intervals $I_{q}$. But by (3)

$$
-1 \leqq \lim \inf \delta(r) \leqq 0 .
$$

Since $\delta(r)$ has local minima at the $r \in M$, there is a $\mu=\mu_{q}^{*} \in I_{q}$ such that

$$
-1 \leqq \delta\left(\mu_{q}^{*}\right) \leqq 0 ; \quad \lim \inf \delta(r)=\lim \inf \delta\left(\mu_{q}^{*}\right)
$$

Since

$$
\delta\left(h_{q}\right)-\delta\left(\mu_{q}^{*}\right)=\log \mu_{q}^{*} / h_{q}-\sum_{h_{q} \leq \mu<\mu_{\dot{q}}^{*}} 2 / \mu \geqq q-2
$$

Similarly

$$
\mu_{q}^{*}>3 h_{q} \quad(q \geqq 4) \text {. }
$$

Now let $g_{1}(z)$ be defined by (1) with $M$ in place of $\Lambda$. As in case (iii) we derive a function

$$
\psi(z)=\sum_{\mu \in M} r_{\mu} z^{\mu-1 / 2}
$$

from the auxiliary function $h(\zeta)=(D \zeta)^{5} / g(\zeta)$. This function $\psi(z)$ has the following properties:

1. Its power series has radius of convergence 1 .

2. As $\mu \rightarrow \infty$ through the sequence $\mu_{1}^{*}, \mu_{2}^{*}, \cdots$,

$$
\lim \sup \left|r_{\mu}\right|^{1 /(\mu-1 / 2)}=1 \text {. }
$$

3. $\psi(z)$ is regular in $x>0$.

4. Consecutive terms of the series have opposite signs.

5. The sign of $\epsilon_{\mu} r_{\mu}$ is the same for all $\mu$ from one and the same interval $I_{q}$.

All these properties are proved as in case 3 .

Now let $n_{q}$ be the largest integer not exceeding $\mu_{q}^{*} / 2$. For any choice of signs

$$
\chi(z)=\sum_{q=4}^{\infty} \pm\left(z(1+z)^{2} / 4\right)^{n_{q}}=\sum c_{n} z^{n}
$$

is regular in the domain $\left|z(1+z)^{2}\right|<4$ which contains $|z| \leqq 1, z \neq 1$. The coefficient $c_{n}$ is 0 , whenever $n$ is not in one of the intervals 
$n_{q} \leqq n \leqq 3 n_{q}$. By (5) and (6) this implies that $c_{n}=0$ for $n \notin I_{q}$ $(q=1,2, \cdots)$. All the nonzero $c_{n}$ whose indices are in a fixed interval $I_{q}$ have the same sign. As $n \rightarrow \infty$ through the sequence $\mu_{1}^{*}, \mu_{2}^{*}, \cdots$, $\left|c_{n}\right|^{1 / n} \rightarrow 1$. This shows that $\chi(z)$ has radius of convergence 1 and so $z=1$ is the only singularity of $\chi(z)$ on the unit circle. The function formed by Hadamard multiplication of $\chi(z)$ and $\psi(z)$, i.e. $\sum_{\mu \in M} r_{\mu} c_{\mu-1 / 2} z^{\mu-1 / 2}=f(z)$ is therefore analytic at all points of $|z|=1$ at which $\psi$ is analytic, i.e. on $|z|=1, x>0$. If the \pm signs in the definition of $\chi(z)$ are chosen suitably, it follows from property 5 of $\psi(z)$ that the coefficient of $z^{\mu-1 / 2}$ in $f(z)$ and $\epsilon_{\mu-1 / 2}$ are of the same sign, since the power series of $f(z)$ contains no terms whose indices are not in one of the intervals $I_{q}$. By adding an entire function to $f(z)$, we can form a power series without vanishing terms which satisfies all requirements.

4. The following example shows that there are sequences $\left\{\epsilon_{k}\right\}$ such that every closed semi-circle on the circle of convergence of $\sum \epsilon_{k} a_{k} z^{k}\left(a_{k}>0\right)$ contains at least one singularity.

Let

$$
\begin{array}{rlrl}
\epsilon_{k} & =1, & k & \equiv 0,1(\bmod 4), \\
\epsilon_{k}=-1, & k & \equiv 2,3(\bmod 4) .
\end{array}
$$

Then $F(z)=\sum \epsilon_{k} a_{k} z^{k}\left(a_{k}>0, \lim \sup a_{\mathbf{k}}^{1 / k}=1\right)$ is a power series whose sequence of coefficients has sign-changes with density $1 / 2$. By a theorem of Pólya (see $[1$, p. 51]) this implies that $F(z)$ has a singularity on $|z|=1,|\arg z| \leqq \pi / 2$. But $F(-z)$ is again a function whose power series coefficients have sign-changes of density $1 / 2$. Therefore Pólya's theorem shows that $F(z)$ has a singularity on $|z|=1$, $|\arg (-z)| \leqq \pi / 2$. Since $F$ has real coefficients, the singularities of $F$ are symmetrically situated with respect to the real axis. It is now easy to see that $F$ has singularities on every closed semi-circle on $|z|=1$.

\section{REFERENCES}

1. L. Bieberbach, Analytische Fortsetzung, Berlin, 1955.

2. W. H. J. Fuchs, On the closure of $\left\{e^{-t a v}\right\}$, Proc. Cambridge Philos. Soc. vol. 42 (1945) pp. 91-105.

3. A. Hurwitz and G. P6lya, Zwei Beweise eines von Herrn Fatou vermuteten Satzes, Acta Math. vol. 40 (1917) pp. 179-181.

Cornell University 